\title{
Antibiotic Resistance Patterns and Distribution of Ampc $\beta$-Lactamases Genes Among Acinetobacter Baumannii Clinical Isolates from Hospitals of Tehran, Iran
}

\section{Sajad Alizadeh}

Islamic Azad University

Majid Baserisalehi ( $D$ Baserisalehi@yahoo.com )

Islamic Azad University

Nima Bahador

Islamic Azad University

\section{Research Article}

Keywords: Acinetobacter baumannii, antibiotic resistance, AmpC $\beta$-lactamases, Tehran.

Posted Date: July 7th, 2021

DOl: https://doi.org/10.21203/rs.3.rs-614798/v1

License: () (1) This work is licensed under a Creative Commons Attribution 4.0 International License.

Read Full License 


\section{Abstract \\ Background}

Acinetobacter is a Gram-negative coccobacilli bacterium that can produce severe and different infections. Among the species of these bacteria, Acinetobacter baumannii is the most common cause of nosocomial infections. Due to the high prevalence of antibiotic resistance in this bacterium and the significant increase in antibiotic resistance, this study was conducted to investigate the antibiotic susceptibility of $A$. baumannii isolates from Iran. A total of $60 \mathrm{~A}$. baumannii bacteria were isolated from the different clinical samples in hospitals of Tehran, Iran. The isolates susceptibility to 13 commonly used antibiotics was examined according to the Clinical \& Laboratory Standards Institute (CLSI) guidelines. Using PCR, three important AmpC $\beta$-lactamases relayed genes (DHA, CIT and MOX) were detected.

\section{Results}

The highest and lowest resistance rate was related to ampicillin (98.3\%) colistin (35\%), respectively. Of 60 isolates, 59 isolates $(98.34 \%$ ) were resistant to more than 8 antibiotics. The frequencies of DHA, CIT and MOX genes were 1 (2\%), 7 (12\%), 27 (46\%), respectively. Based on definition, 59 (88.33\%), 44 (73.33\%) and $13(21.66 \%)$ isolates were MDR, XDR and PDR, respectively. Twenty-four isolates (40\%) were negative for all three genes. There was a significant relationship between the presence of MOX gene and antibiotic resistance.

\section{Conclusions}

The high resistance rates of the $A$. baumannii isolates reported in the present study is alarming and need the management of treatment such as performing of antibiogram test before antibiotic therapy for select the appropriate antibiotic and also completes the course of treatment period.

\section{Background}

Acinetobacter is a non-motile, ubiquitous Gram-negative bacterium with the ability to grow on different laboratory culture media [1]. This opportunistic pathogen can colonize on the skin and mucosal membranes of the respiratory system of infected individuals [2]. Different species of this genus can cause various nosocomial infections, commonly among patients in intensive care units (ICUs) and highdependency units (HDUs) [3]. Acinetobacter baumannii is more prevalent than other strains of genus Acinetobacter, and it is the most common cause of bacterium-induced infections [4]. Ventilatorassociated pneumonia (VAP), septicemia, secondary meningitis, endocarditis, infections of the skin, soft tissues, urinary tract, and wound infection can be caused by bacteria A. baumannii bacteria [5]. 
To treatment of $A$. baumannii infections, various antibiotics, including $\beta$-lactams, fluoroquinolones, and aminoglycosides, are used. Today, many studies have shown that antibiotic resistance in the $A$. baumannii isolates is extremely increasing $[3,4,6,7]$. Inappropriate and indiscriminate use of available antibiotics, high ability of natural genetic transformation in $A$. baumannii bacteria and its potential for widespread dissemination are most important factors contributed to the resistance of this pathogen to the various antimicrobial agents $[8,9]$.

Currently, $\beta$-lactam antibiotics play critical roles in the treatment of $A$. baumannii infections. But from 1991 until now there are many studies that reported the resistance of $A$. baumannii to this class of antibiotics [5]. Molecularly, resistance to $\beta$-lactams in A. baumannii may be due to the activity efflux pumps, changes in penicillin-binding proteins (PBPs), and the production of antibiotic-degrading enzymes such as production of $\beta$-lactamases [10].

Members of Gram-negative bacteria can hydrolyze many $\beta$-lactam antibiotics through the production of one or both of extended-spectrum $\beta$-lactamases (ESBLs) and AmpC $\beta$-lactamases [11]. AmpC $\beta$ lactamases are clinically significant because they may confer resistance to a wide variety of $\beta$-lactam antibiotics, including a-methoxy- $\beta$-lactams, such as cefoxitin, narrow-, expanded-, and broad-spectrum cephalosporins, $\beta$-lactam- $\beta$-lactamase-inhibitor combinations, and aztreonam. AmpC $\beta$-lactamases are poorly inhibited by clavulanic acid; however, they are inhibited by cloxacillin [12]. AmpC $\beta$-lactamases is encoded by chromosomal or plasmid genes. Plasmid-mediated include MOX-, CIT-, DHA-, ACC-, FOX-, and EBC-type enzymes, and CMY-2 of CIT-type enzymes has shown the broadest geographic spread and is one of the main causes of $\beta$-lactam resistance at present [13].

According to these facts the present study was aimed to evaluate the phenotypic and genotypic antibiotic resistance pattern of $A$. baumannii clinical isolates and comparison of results to the previous described findings. In addition, we investigated the presence of AmpC $\beta$-lactamases enzyme production and also AmpC $\beta$-lactamases related genes.

\section{Results}

\section{Demographic data}

A total of $60 \mathrm{~A}$. baumannii isolates were isolated from 240 clinical isolates were obtained based on applied differential and biochemical tests. These isolates were obtained from 27 (45\%) women and 33 (55\%) men. Among them, 6 (10\%) cases hospitalized due to abdominal surgery, 7 (11.66\%) cases due to heart surgery, 2 (3.33) cases due to brain surgery and 11 (18.33\%) cases due to respiratory problems. These data are presented in detail in Table 1. 
Table 1

Clinical characteristics of patients infected with $A$. baumannii isolates

\begin{tabular}{|llll|}
\hline Cause of hospitalization & Women & Men & Total \\
\hline abdominal surgery & $2(3.33 \%)$ & $4(6.66 \%)$ & $6(10 \%)$ \\
\hline heart surgery & $3(5 \%)$ & $4(6.66 \%)$ & $7(11.66 \%)$ \\
\hline brain surgery & $1(1.66 \%)$ & $1(1.66 \%)$ & $2(3.33 \%)$ \\
\hline respiratory problems & $4(6.66 \%)$ & $7(11.66 \%)$ & $11(18.33 \%)$ \\
\hline heart problems & $3(5 \%)$ & $6(10 \%)$ & $9(15 \%)$ \\
\hline cancer & $2(3.33 \%)$ & $6(10 \%)$ & $8(13.33 \%)$ \\
\hline premature infant & $1(1.66 \%)$ & $1(1.66 \%)$ & $2(3.33 \%)$ \\
\hline stroke & 0 & $2(3.33 \%)$ & $2(3.33 \%)$ \\
\hline diabetes & $1(1.66 \%)$ & $1(1.66 \%)$ & $2(3.33 \%)$ \\
\hline pelvic surgery & $1(1.66 \%)$ & $1(1.66 \%)$ & $2(3.33 \%)$ \\
\hline brain problems & $3(5 \%)$ & 0 & $3(5 \%)$ \\
\hline plastic surgery and liposuction & $4(6.66 \%)$ & 0 & $4(6.66 \%)$ \\
\hline aortic valve surgery & $1(1.66 \%)$ & 0 & $1(1.66 \%)$ \\
\hline parturition & $1(1.66 \%)$ & 0 & $1(1.66 \%)$ \\
\hline Total & $27(45 \%)$ & $33(55 \%)$ & $60(100 \%)$ \\
\hline
\end{tabular}

\section{Antibiotic resistance patterns}

Our results revealed that out of 15 studied antibiotics, the resistance rate above $90 \%$ was reported in 12 cases. Based on our results the tested isolates are highly resistant to the ampicillin (99\%), cefotaxime (98\%), chloramphenicol (98\%), ceftriaxone (98\%), ceftazidime (98\%), meropenem (98\%) and ticarcillin (98\%). On the other hand, tobramycin and colistin with $78.3 \%$ and $35 \%$ resistance rates were the most effective antibiotics, respectively. The results of the present study showed high resistance rate of clinical isolates to tested antibiotics. The total resistance pattern was presented in Table 2 . In this study, out of 60 isolates, 59 isolates (98.34\%) were resistant to more than 8 antibiotics and only 1 sample (1.66\%) which isolated from urine culture was sensitivity to all studied antibiotics. 
Table 2

Antibiotic resistance pattern of $A$. baumannii isolates

\begin{tabular}{|lllll|}
\hline Antimicrobial category & Antimicrobial agent & \multicolumn{3}{l}{ Number (Frequency) } \\
\cline { 2 - 5 } & & $\mathbf{R}$ & $\mathbf{I}$ & $\mathbf{S}$ \\
\hline Aminoglycosides & gentamicin & $57(95)$ & 0 & $3(0.5)$ \\
& amikacin & $47(78.3)$ & 0 & $13(21.7)$ \\
\cline { 2 - 5 } & tobramycin & $47(78.3)$ & 0 & $13(21.7)$ \\
\hline Cephalosporins & cefotaxime & $58(96.7)$ & 0 & $2(3.3)$ \\
\cline { 2 - 5 } & ceftriaxone & $58(96.7)$ & 0 & $2(3.3)$ \\
\cline { 2 - 5 } & ceftazidime & $58(96.7)$ & $1(1.7)$ & $1(1.7)$ \\
\cline { 2 - 5 } & cefepime & $55(90)$ & $1(1.7)$ & $4(6.7)$ \\
\hline Carbapenems & meropenem & $58(96.7)$ & 0 & $2(3.3)$ \\
\hline Penicillins & ampicillin & $59(98.3)$ & 0 & $1(1.7)$ \\
\cline { 2 - 5 } & piperacillin & $55(90)$ & $1(1.7)$ & $4(6.7)$ \\
\cline { 2 - 5 } & ticarcillin & $58(96.7)$ & 0 & $2(3.3)$ \\
\hline Fluoroquinolones & ciprofloxacin & $56(93.3)$ & 0 & $4(6.7)$ \\
\hline Sulfonamide & Trimethoprim/sulfamethoxazole & $56(93.3)$ & 0 & $4(6.7)$ \\
\hline Chloramphenicol & chloramphenicol & $58(96.7)$ & 0 & $2(3.3)$ \\
\hline Polymyxins & colistin & $21(35)$ & $2(3.3)$ & \\
\hline
\end{tabular}

\section{MDR, XDR and PDR isolates}

Based on definition of tested isolates, 59 isolates (88.33\%) were in the MDR group, where 44 isolates (73.33\%) were XDR. Our results showed that 13 isolates (21.66\%) were PDR.

\section{Distribution of Amp $\beta$-lactamases genes}

The prevalence of DHI, MOX and CIT were 1 (1.66\%), 27 (45\%) and 7 (11.66\%), respectively. Twenty-four isolates $(40 \%)$ were negative for all three genes. There was a significant relationship between the presence of MOX gene and antibiotic resistance $(p<0.05)$. In other word, MOX-containing isolates were resistance to more tested antibiotics.

\section{Discussion}


In the past 2 decades, $A$. baumannii has become an important pathogen related to nosocomial infections and has been shown to increase morbidity and mortality. Control of infections caused by this bacteria is always difficult, due to its acquisition of multidrug-resistant phenotypes, such as resistance to fluoroquinolones, aminoglycosides, cephalosporins, and carbapenems [1].

In the present study, the antibiotic resistance profile as well as the phenotypic and genotypic AmpC $\beta$ lactamases resistance was investigated. Our results revealed that out of 15 studied antibiotics, 12 antibiotics had a resistance above $90 \%$. The most resistance rate was related to ampicillin (99\%), cefotaxime (98\%), chloramphenicol (98\%), ceftriaxone (98\%), ceftazidime (98\%), meropenem (98\%) and ticarcillin (98\%). These results can be more important in related to the antibiotics which are used to treatment of $A$. baumannii infections; such as third- and fourth-generation cephalosporins and carbapenems [2].

The results of this study are in line with the findings of previous studies which reported that most strains of $A$. baumannii isolated in Iran were resistant to first-line drugs including aminoglycosides, fluoroquinolones and carbapenems [14-16]. In a study conducted by Karimi et al (2020) on $60 \mathrm{~A}$. baumannii isolates obtained from Hazrat-e-Rasoul Hospital in Tehran, the highest resistance of isolates was related to ceftazidime (93.3\%) and amikacin (91.6\%), respectively. In their study, colistin with 3.3\% resistance was introduced as the most effective antibiotic [17]. In terms of antibiotic resistance profile, the results of this study were in line with our present study.

Due to rising antimicrobial resistance, carbapenems are the cornerstone of therapy for the treatment of $A$. baumannii serious infections [18]. However, the results of the present study showed a high prevalence of meropenem resistance $(96.7 \%)$, which has increased significantly compared to previous studies in different cities of Iran and also other countries $[19,20]$. In similar studies in Tehran, $50.9 \%, 52.5 \%, 62 \%$ and $67.5 \%$ of the isolates were resistant to imipenem and $51.8 \%, 52.5 \%, 62 \%$ and $84.5 \%$ to meropenem in 2008, 2009, 2011 and 2013, respectively [16, 21-23].

The analysis of these data and comparing them with the results of the present study results reveals that antibiotic resistance of $A$. baumannii isolates to carbapenem antibiotics were increasing in Iran over the time.

In our study low susceptibility rates to most of available antimicrobial agents for the treatment of $A$. baumannii isolates was seen, except for colistin. Although several number of previous studies conducted in Iran have shown that all of the $A$. baumannii isolates were sensitive to colistin [15, 21, 24], but in the present study, $35 \%$ of the isolates were resistant to colistin. Given that drug resistance rate of $A$. baumannii to colistin was not high, this antibiotic can be administered as appropriate therapeutic drug against $A$. baumannii infections.

Based on our results, 59 (88.33\%), 44 (73.33\%) and 13 (21.66\%) isolates were MDR, XDR and PDR, respectively. Based on studies, emergence of MDR, XDR and PDR of $A$. baumannii strain are currently reported [25]. 
In contrast to the present study which found PDR and XDR as well as MDR A. baumannii isolates, Hojabri (2014) only found MDR strains among the isolates [26]. In Bahador et al (2015) study, the frequency of MDR, XDR and PDR isolates were 69, 24, and 0\%, respectively[27]. In similar study performed by Sobouti et al (2020) in Iran, from 62 A. baumannii, 36 (58\%) strains were categorized as MDR, 17 (27.5\%) as XDR, and 9 (14.5\%) as PDR [25]. Compared to the results of this study, the percentage of resistant strains in the present study was higher. Comparison of these results and also similar studies shows that the emergence of resistant strains in $A$. baumannii isolates is increasing in Iran and the strict control measures should be considered in this subject.

In next part of study the prevalence of some AmpC $\beta$-lactamases related genes (FOX, DHA and MOX) in $A$. baumannii isolates were detected. Based on these results, it was found that out of 60 isolates, 1 (1.66\%), 27 (45\%) and 7 (11.66\%) isolates were positive for DHI, MOX and CIT, respectively. Consistent with the present study, in Fekri et al (2017) study AmpC $\beta$-lactamases related genes detected in 60 strains $A$. baumannii isolates using Multiplex PCR, of which 39 (65\%) had CIT, 36 (60\%) had DHA and 12 (20\%) had MOX gene [28]. The products of these genes were consistently associated with resistance or at least reduced susceptibility to a wide variety of $\beta$-lactam antibiotics. For this reason the presence of these genes in the individual isolate is very important [29]. Our results also shown that, there is some cephalosporins-resistant isolates which are negative for the some investigated genes. Resistance to these antibiotics in those isolates may be due to other mechanisms including decreased permeability, alteration of PBPs, presence of other AmpC related genes and overexpression of efflux pumps [30].

\section{Conclusion}

This study has shown that resistance to the majority of antibiotics in the $A$. baumannii strains is high with 59 (88.33\%), 44 (73.33\%) and 13 (21.66\%) isolates were MDR, XDR and PDR, respectively. High rate of resistance to cephalosporins has been seen among our isolates and it seems that, colistin can be effective drug for treatment of $A$. baumannii infections. Progressive increase in resistance to the majority of antibiotics and multiple resistances in the present study may be related to increased usage of these antibiotics for treatment of $A$. baumannii infections. Management of treatment, such as performing of antibiogram test before antibiotic therapy for select the appropriate antibiotic and also completes the course of treatment period is necessary to prevent the further spread of resistant isolates.

\section{Methods}

\section{Bacterial isolation}

A total of 240 clinical samples (including blood, urine, sputum, respiratory secretions, urine, wounds and skin) were collected from patients admitted to different wards of hospitals in Tehran between 2018 to 2020. Samples were collected from patients who had been hospitalized for at least three days and who had received the infection from the hospitals. A. baumannii were identified using Gram staining, culture characteristics on the differential culture media (blood agar, MacConkey agar, Triple sugar iron agar (TSI) 
and oxidative-fermentative (OF) agar and biochemical tests (catalase and oxidase) [31]. The isolated bacteria were stored in Tryptic Soy Broth (TSB; Merck, Germany) containing $30 \%$ glycerol at $-70^{\circ} \mathrm{C}$ until further analysis.

\section{Antibiotic Susceptibility Testing}

The antibiotic susceptibilities testing for $A$. baumannii isolates were done by Kirby Bauer's disk diffusion method on Muller-Hinton agar (Merk, Germany) according to the CLSI [32]. The applied antibiotic disks (MAST, UK) were ampicillin $(25 \mu \mathrm{g})$, cefotaxime $(30 \mu \mathrm{g})$, chloramphenicol $(30 \mu \mathrm{g})$, ceftriaxone $(30 \mu \mathrm{g})$, ceftazidime $(30 \mu \mathrm{g})$, meropenem $(10 \mu \mathrm{g})$, ticarcillin $(75 \mu \mathrm{g})$, gentamicin $(30 \mu \mathrm{g})$, ciprofloxacin $(5 \mu \mathrm{g})$, trimethoprim/sulfamethoxazole $(75 \mu \mathrm{g})$, cefepime $(30 \mu \mathrm{g})$, piperacillin $(100 \mu \mathrm{g})$, amikacin $(30 \mu \mathrm{g})$, tobramycin $(30 \mu \mathrm{g})$, colistin $(25 \mu \mathrm{g})$.

\section{Determination of multidrug-resistant (MDR), extensively drug-resistant (XDR) and pandrug-resistant (PDR) isolates}

According to the CLSI, MDR was defined as acquired non-susceptibility to at least one agent in three or more antimicrobial categories, XDR was defined as non-susceptibility to at least one agent in all but two or fewer antimicrobial categories for example bacterial isolates remain susceptible to only one or two categories and PDR was defined as non-susceptibility to all agents in all antimicrobial [33].

\section{Dna Extraction And Multiplex Pcr}

DNA extraction was carried out from $10 \mathrm{ml}$ overnight cultures of each isolate in tryptic soy broth (TSB) by Cinnagen extraction kit (Cinnagen, Iran) according to the manufactory instructions. Extracted DNA samples from all of the isolates were examined by the PCR assay for three target genes, MOX, CIT and DHA.

The oligonucleotide primers were previously described by Hanson et al [34] and their sequences are given in Table 3. For each gene, the final volume of the reaction mixture was $25 \mu$ contained $3 \mu \mathrm{l}$ of template DNA, $2.5 \mu \mathrm{l}$ of $\times 10$ PCR buffer, $0.5 \mu \mathrm{l}$ of $10 \mathrm{mM}$ dNTPs, $0.75 \mu \mathrm{l}$ of $50 \mathrm{mM} \mathrm{MgCl} 2,0.25 \mu \mathrm{l}$ of $5 \mathrm{U} / \mu \mathrm{l}$ of Taq DNA polymerase, and $25 \mathrm{pmol}$ of each used primer.

The PCR amplification was performed under the following thermal conditions: initial denaturation at $94^{\circ} \mathrm{C}$ for $1 \mathrm{~min}$ followed by denaturation at $94^{\circ} \mathrm{C}$ for $1 \mathrm{~min}$, annealing at $54^{\circ} \mathrm{C}$ for $1 \mathrm{~min}$ and extension at $72^{\circ} \mathrm{C}$ for $1.5 \mathrm{~min}$ ( $30 \mathrm{cycles}$ ), and a final extension at $72^{\circ} \mathrm{C}$ for $10 \mathrm{~min}$. The PCR products were analyzed by electrophoresis on $1.5 \%$ agarose gel containing $0.5 \mu \mathrm{g} / \mathrm{ml}$ ethidium bromide. 
Table 3

Sequence of primers used to screen studied genes

\begin{tabular}{|c|c|c|c|c|}
\hline Target & Primer & Sequence ( $5^{\prime}$ to $\left.3^{\prime}\right)$ & Size (bp) & Reference \\
\hline \multirow[t]{2}{*}{ MOX } & MOX $-\mathrm{F}$ & GCTGCTCAAGGAGCACAGGAT & \multirow[t]{2}{*}{520} & \multirow[t]{6}{*}{ [34] } \\
\hline & MOX $-\mathrm{R}$ & CACATTGACATAGGTGTGGTGC & & \\
\hline \multirow[t]{2}{*}{$C I T$} & CIT-F & TGGCCAGAACTGACAGGCAAA & \multirow[t]{2}{*}{462} & \\
\hline & CIT-R & TTTCTCCTGAACGTGGCTGGC & & \\
\hline \multirow[t]{2}{*}{$D H A$} & DHA-F & AACTTTCACAGGTGTGCTGGGT & \multirow[t]{2}{*}{246} & \\
\hline & DHA-R & CCGTACGCATACTGGCTTTGC & & \\
\hline
\end{tabular}

\section{Abbreviations}

PCR: Polymerase chain reaction; $A$. baumannii: Acinetobacter baumannii; CLSI: Clinical \& Laboratory Standards Institute; MDR: Multidrug-resistant; XDR: Extensively drug-resistant PDR: Pandrug-resistant; ICU: Intensive care units; HDU: High-dependency units; VAP: Ventilator-associated pneumonia; PBPs: Penicillin-binding proteins; ESBLs: extended-spectrum $\beta$-lactamases; TSI: Triple sugar iron agar; OF: Oxidative-fermentative; TSB: Tryptic soy broth.

\section{Declarations}

\section{Acknowledgments}

The authors would like to thank Kazeroun branch of Islamic Azad University.

\section{Authors' contributions}

MB designed the study wrote the manuscript. SA does laboratory tests. NB analysis the results. All authors read and approved the manuscript.

\section{Funding}

No funding was obtained for this study.

\section{Availability of data and materials}

All data generated during this study are included in this published article.

\section{Ethics approval and consent to participate}

\section{Ethics statement}


This research was approved by the Ethical Committee of Islamic Azad University, Kazerun Branch, Kazerun, Iran (Code No: IR.IAU.KAU.REC.1399.104). In addition, informed consent form was obtained from all of the patients.

\section{Consent for publication}

Not Applicable.

\section{Competing interests}

The authors declare that they have no competing interests.

\section{References}

1. Houang ET, Chu Y, Leung C, Chu K, Berlau J, Ng K, et al. Epidemiology and Infection Control Implications ofAcinetobacter spp. in Hong Kong. Journal of clinical microbiology 2001;39:228-34.

2. Villers D, Espaze E, Coste-Burel M, Giauffret F, Ninin E, Nicolas F, et al. Nosocomial Acinetobacter baumannii infections: microbiological and clinical epidemiology. Annals of internal medicine 1998;129:182-9.

3. Michalopoulos A, Falagas ME. Treatment of Acinetobacter infections. Expert opinion on pharmacotherapy 2010;11:779-88.

4. Azizi M, Mortazavi SH, Etemadimajed M, Gheini S, Vaziri S, Alvandi A, et al. Prevalence of ExtendedSpectrum $\beta$-Lactamases and Antibiotic Resistance Patterns in Acinetobacter baumannii Isolated from Clinical Samples in Kermanshah, Iran. Jundishapur Journal of Microbiology 2017;10:1-7.

5. Levin AS, Levy CE, Manrique AEI, Medeiros EA, Costa SF. Severe nosocomial infections with imipenem-resistant Acinetobacter baumannii treated with ampicillin/sulbactam. International journal of antimicrobial agents 2003;21:58-62.

6. Ellis D, Cohen B, Liu J, Larson E. Risk factors for hospital-acquired antimicrobial-resistant infection caused by Acinetobacter baumannii. Antimicrobial resistance and infection control 2015;4:1-5.

7. Dagher TN, Al-Bayssari C, Chabou S, Antar N, Diene SM, Azar E, et al. Investigation of multidrugresistant ST2 Acinetobacter baumannii isolated from Saint George hospital in Lebanon. BMC microbiology 2019;19:1-7.

8. Magiorakos AP, Srinivasan A, Carey R, Carmeli Y, Falagas M, Giske C, et al. Multidrug-resistant, extensively drug-resistant and pandrug-resistant bacteria: an international expert proposal for interim standard definitions for acquired resistance. Clinical microbiology and infection 2012;18:268-81.

9. Tada T, Uchida H, Hishinuma T, Watanabe S, Tohya M, Kuwahara-Arai K, et al. Molecular epidemiology of multidrug-resistant Acinetobacter baumannii isolates from hospitals in Myanmar. Journal of Global Antimicrobial Resistance 2020.

10. Liu Y, Liu X. Detection of AmpC $\beta$-lactamases in Acinetobacter baumannii in the Xuzhou region and analysis of drug resistance. Experimental and therapeutic medicine 2015;10:933-6. 
11. Ibrahim ME, Abbas M, Al-Shahrai AM, Elamin BK. Phenotypic characterization and antibiotic resistance patterns of extended-spectrum $\beta$-Lactamase-and AmpC $\beta$-lactamase-producing Gramnegative bacteria in a referral hospital, Saudi Arabia. Canadian Journal of Infectious Diseases and Medical Microbiology 2019;2019.

12. Shahid M, Malik A, Agrawal M, Singhal S. Phenotypic detection of extended-spectrum and AmpC $\beta$ lactamases by a new spot-inoculation method and modified three-dimensional extract test: comparison with the conventional three-dimensional extract test. Journal of Antimicrobial Chemotherapy 2004;54:684-7.

13. Ye Q, Wu Q, Zhang S, Zhang J, Yang G, Wang H, et al. Antibiotic-resistant extended spectrum sslactamase-and plasmid-mediated AmpC-producing enterobacteriaceae isolated from retail food products and the pearl river in Guangzhou, China. Frontiers in microbiology 2017;8:96.

14. Karbasizade V, Heidari L, Jafari R. Detection of OXA-Type Carbapenemase Genes in Acinetobacter baumannii Isolates from Nosocomial Infections in Isfahan Hospitals, Iran. Journal of Medical Bacteriology 2015;4:31-6.

15. Shoja S, Moosavian M, Peymani A, Tabatabaiefar MA, Rostami S, Ebrahimi N. Genotyping of carbapenem resistant Acinetobacter baumannii isolated from tracheal tube discharge of hospitalized patients in intensive care units, Ahvaz, Iran. Iranian journal of microbiology 2013;5:315.

16. Feizabadi M, Fathollahzadeh B, Taherikalani M, Rasoolinejad M, Sadeghifard N, Aligholi M, et al. Antimicrobial susceptibility patterns and distribution of blaOXA genes among Acinetobacter spp. Isolated from patients at Tehran hospitals. Jpn J Infect Dis 2008;61:274-8.

17. Karimi F, Amini K, Javadi G. A Phenotypic and Genotypic Study of Colistin arn Resistance Regulator Gene Classes in Acinetobacter Baumannii isolated from Clinical Cases using Multiplex PCR.

18. Evans SR, Hujer AM, Jiang H, Hill CB, Hujer KM, Mediavilla JR, et al. Informing antibiotic treatment decisions: evaluating rapid molecular diagnostics to identify susceptibility and resistance to carbapenems against Acinetobacter spp. in PRIMERS III. Journal of clinical microbiology 2017;55:134-44.

19. Dauner D, May J, Steele J. Assessing antibiotic therapy for Acinetobacter baumannii infections in an academic medical center. European journal of clinical microbiology \& infectious diseases 2008;27:1021.

20. Keikha M, Jadidi H. Meta-analysis study for antibiotic resistance of Acinetobacter baumannii clinical isolates. Iranian Journal of Medical Microbiology 2017;11:69-74.

21. Karmostaji A, Peerayeh SN, Salmanian AH. Distribution of OXA-type class D $\beta$-lactamase genes among nosocomial multi drug resistant Acinetobacter baumannii isolated in Tehran hospitals. Jundishapur Journal of Microbiology 2013;6.

22. Sohrabi N, Farajnia S, Akhi MT, Nahaei MR, Naghili B, Peymani A, et al. Prevalence of OXA-type $\beta$ lactamases among Acinetobacter baumannii isolates from Northwest of Iran. Microbial Drug Resistance 2012;18:385-9. 
23. Morovat T, Bahram F, Mohammad E, Setareh S, Mohamad Mehdi F. Distribution of different carbapenem resistant clones of Acinetobacter baumannii in Tehran hospitals. The new microbiologica 2009;32:265.

24. Kooti S, Motamedifar M, Sarvari J. Antibiotic resistance profile and distribution of oxacillinase genes among clinical isolates of Acinetobacter baumannii in Shiraz teaching hospitals, 2012-2013. Jundishapur journal of microbiology 2015;8.

25. Sobouti B, Mirshekar M, Fallah S, Tabaei A, Mehrabadi JF, Darbandi A. Pan drug-resistant Acinetobacter baumannii causing nosocomial infections among burnt children. Medical journal of the Islamic Republic of Iran 2020;34:24.

26. Hojabri Z, Pajand O, Bonura C, Aleo A, Giammanco A, Mammina C. Molecular epidemiology of Acinetobacter baumannii in Iran: endemic and epidemic spread of multiresistant isolates. Journal of Antimicrobial Chemotherapy 2014;69:2383-7.

27. Bahador A, Raoofian R, Pourakbari B, Taheri M, Hashemizadeh Z, Hashemi FB. Genotypic and antimicrobial susceptibility of carbapenem-resistant Acinetobacter baumannii: analysis of ISAba elements and blaOXA-23-like genes including a new variant. Frontiers in microbiology 2015;6:1249.

28. Fekri S, Soltani Banavandi MJ, Amini M. Molecular Study of Plasmid Genes Ampc of Acinetobacter baumannii Isolated from Clinical Cases Using Multiplex PCR. Journal of Mazandaran University of Medical Sciences 2017;27:157-62.

29. Kumar E, Usha K, Chaudhury A, Ramana B, Gopal DS. Detection of AmpC $\beta$-lactamases production in Acinetobacter species by inhibitor (disk) based \& modified three dimensional (enzyme extraction) methods. The Indian journal of medical research 2014;140:688.

30. Livermore DM. Mechanisms of resistance to cephalosporin antibiotics. Drugs 1987;34:64-88.

31. Héritier C, Poirel L, Fournier P-E, Claverie J-M, Raoult D, Nordmann P. Characterization of the naturally occurring oxacillinase of Acinetobacter baumannii. Antimicrobial agents and chemotherapy 2005;49:4174-9.

32. Goudarzi H, Douraghi M, Ghalavand Z, Goudarzi M. Assessment of antibiotic resistance pattern in Acinetobacter bumannii carrying bla oxA type genes isolated from hospitalized patients. Novelty in Biomedicine 2013;1:54-61.

33. Magiorakos A-P, Srinivasan A, Carey R, Carmeli Y, Falagas M, Giske C, et al. Multidrug-resistant, extensively drug-resistant and pandrug-resistant bacteria: an international expert proposal for interim standard definitions for acquired resistance. Clinical microbiology and infection 2012;18:268-81.

34. Hanson NJD, Perez F-JP. Multiplex PCR for the detection of AmpC beta-lactamase genes. Google Patents; 2006. 